\title{
HARNACK'S INEQUALITY IN THE BORDERLINE CASE
}

\author{
SEPPO GRANLUND
}

\section{Introduction}

Harnack's inequality for general quasi-linear elliptic equations has been proved by Serrin [13] and Trudinger [14]. In both papers the proof is based on the iteration method introduced by Moser in [7] and [8]. In this method the lemma of John and Nirenberg [4] is essential.

We consider variational integrals of the form

$$
I(u)=\int_{G} F(x, \nabla u(x)) d m(x),
$$

where $G \subset R^{n}$ is a bounded domain, $u \in W_{n}^{1}(G)$, and the kernel $F: G \times R^{n} \rightarrow R$ satisfies the following conditions:

(1.2) The functions $x \mapsto F(x, \nabla u(x))$ are measurable for all $u \in W_{n}^{1}(G)$.

(1.3) For a.e $x \in G$ the function $z \rightarrow F(x, z)$ is convex and $\alpha|z|^{n} \leqq F(x, z) \leqq \beta|z|^{n}$ for all $z \in R^{n}$, where $\alpha, \beta>0$ are constants.

Let $\varphi \in W_{n}^{1}(G)$ and write $\mathscr{F}_{\varphi}(G)=\left\{u \in W_{n}^{1}(G) \mid u-\varphi \in W_{n, 0}^{1}(G)\right\}$. A function $u_{0} \in \mathscr{F}_{\varphi}(G)$ is an extremal for the integral (1.1) if $I(u) \geqq I\left(u_{0}\right)$ for all $u \in \mathscr{F}_{\varphi}(G)$. It can be proved that $u_{0}$ is locally Hölder continuous in $G$; see [6, Theorem 4.3.1] and [3, Remark 5.7]. Let $u_{0}$ be an extremal for the integral (1.1) and $u_{0}(x) \geqq 0$ for $x \in G$. Harnack's inequality takes the following form:

1.4. Theorem. Let $\bar{B}^{n}\left(x_{0}, 2 r\right) \subset G$. Then $\max u_{0} \leqq c \min u_{0}$ in $B^{n}\left(x_{0}, r\right)$. The constant $c$ depends only on $\alpha / \beta$ and $n$.

Our proof is based on an oscillation lemma for monotone functions in the space $W_{n}^{1}(G) \cap C(G)$. Lemmas of this type have been proved by Gehring [1, Lemma 1] and Mostow [9, Lemma 4.1]. This makes it possible to avoid the lemma of John and Nirenberg. In the case $n=2$ a similar method has been used earlier for linear elliptic equations; see Gilbarg-Trudinger [2, p. 200]. 


\section{Preliminary lemmas}

We first give the definition of monotone functions.

2.1. Definition. Let $G \subset R^{n}$ be a bounded domain. A function $u \in C(G)$ is monotone in $G$ if

$$
\sup _{x \in D} u(x)=\sup _{x \in \partial D} u(x) \text { and } \inf _{x \in D} u(x)=\inf _{x \in \partial D} u(x)
$$

for all domains $D, \bar{D} \subset G$.

The following lemma gives an estimate for the oscillation of monotone functions in the space $W_{n}^{1}(G)$.

2.2. Lemma. Let $u \in C(G) \cap W_{n}^{1}(G)$ and $B^{n}\left(x_{0}, 2 r\right) \subset G$. If $u$ is monotone in $G$, we have

$$
\underset{B^{n}\left(x_{0}, r\right)}{\operatorname{OSc}}\{u\} \leqq \gamma\left(\int_{B^{n}\left(x_{0}, 3 r / 2\right)}|\nabla u|^{n} d m\right)^{1 / n} .
$$

The constant $\gamma$ depends only on $n$.

Proof. The inequality can be easily derived from the oscillation lemma proved in [9, Lemma 4.1]; see also [1, Lemma 1].

The next lemma gives a weak maximum principle for the extremals of the integral (1.1). In what follows $\varphi \in W_{n}^{\mathbf{1}}(G)$ will be fixed.

2.3. Lemma. Let $u_{0} \in \mathscr{F}_{\varphi}(G)$ be an extremal for the integral (1.1). Then $u_{0}$ is monotone in $G$.

Proof. Suppose that there is a domain $D, \bar{D} \subset G$, and a point $x_{0} \in D$ such that

$$
u_{0}\left(x_{0}\right)>\max _{x \in \partial D} u_{0}(x)=a .
$$

Define $\delta(x)=\inf \left\{u_{0}(x), a\right\}$ for $x \in D$. Then $\delta \in \mathscr{F}_{u_{0}}(D)$ and $\nabla \delta(x)=0$ a.e. on the set $\left\{x \in D \mid u_{0}(x) \geqq a\right\}$. It follows from the condition (1.3) that

$$
\int_{D} F(x, \nabla \delta) d m(x)<\int_{D} F\left(x, \nabla u_{0}\right) d m(x) .
$$

This is a contradiction since $u_{0}$ is an extremal in the class $\mathscr{F}_{u_{0}}(D)$. Thus

$$
\sup _{x \in D} u_{0}(x)=\sup _{x \in \partial D} u_{0}(x) \text {. }
$$

We prove the corresponding equation for minimum values exactly by the same argument. It follows that $u_{0}$ is monotone in $G$.

Let $u_{0} \in \mathscr{F}_{\varphi}(G)$ be an extremal for the integral (1.1) and $u_{0}(x)>0$ for $x \in G$. Define $v(x)=\log u_{0}(x)$ for $x \in G$. 
2.4. Lemma. Let $\bar{B}^{n}\left(x_{0}, 4 r / 3\right) \subset G$. Then

$$
\int_{B^{n}\left(x_{0}, r\right)}|\nabla v|^{n} d m \leqq c_{0} .
$$

The constant $c_{0}$ depends only on $\alpha / \beta$ and $n$.

Proof. We may assume that $u_{0}(x) \geqq(n-1)^{1 / n}$ for $x \in B^{n}\left(x_{0}, 4 r / 3\right)$. If this is not the case we consider the function $\lambda u_{0}$, where $\lambda>0$ is large enough. The function $\lambda u_{0}$ is an extremal for a variational integral, which is of the form (1.1) and satisfies the structure condition (1.3) with the same constants $\alpha$ and $\beta$.

Let $\xi \in C_{0}^{\infty}\left(B^{n}\left(x_{0}, 4 r / 3\right)\right)$ be a non-negative function such that $\xi(x)=1$ for $x \in B^{n}\left(x_{0}, r\right)$ and $|\nabla \xi(x)| \leqq c_{1} / r$. We choose

$$
h(x)=u_{0}(x)+\frac{\xi^{n}(x)}{u_{0}(x)^{n-1}} .
$$

Then $h \in \mathscr{F}_{u_{0}}\left(B^{n}\left(x_{0}, 4 r / 3\right)\right)$, and it has the generalized derivatives

$$
h_{x_{i}}=u_{0 x_{i}}+\frac{n \xi^{n-1}}{u_{0}^{n-1}} \xi_{x_{i}}-(n-1) \frac{\xi^{n}}{u_{0}^{n}} u_{0 x_{i}}=\left(1-(n-1) \frac{\xi^{n}}{u_{0}^{n}}\right) u_{0 x_{i}}+n \frac{\xi^{n-1}}{u_{0}^{n-1}} \xi_{x_{i}} .
$$

Write $S=\left\{x \in B^{n}\left(x_{0}, 4 r / 3\right) \mid \xi(x)=0\right\}$. Suppose that $x \in B^{n}\left(x_{0}, 4 r / 3\right) \backslash S$. The convexity and growth condition (1.3) yields

$$
\begin{aligned}
F(x, \nabla h) & \leqq\left(1-(n-1) \frac{\xi^{n}}{u_{0}^{n}}\right) F\left(x, \nabla u_{0}\right)+(n-1) \frac{\xi^{n}}{u_{0}^{n}} F\left(x, \frac{n}{n-1} \frac{u_{0}}{\xi} \nabla \xi\right) \\
& \leqq\left(1-(n-1) \frac{\xi^{n}}{u_{0}^{n}}\right) F\left(x, \nabla u_{0}\right)+\beta \frac{n^{n}}{(n-1)^{n-1}}|\nabla \xi|^{n} .
\end{aligned}
$$

Since $u_{0}$ is minimizing for the integral (1.1), we get

$$
\begin{aligned}
& \int_{B^{n}\left(x_{0}, 4 r / 3\right)} F\left(x, \nabla u_{0}\right) d m(x) \leqq \int_{B^{n}\left(x_{0}, 4 r / 3\right)} F(x, \nabla h) d m(x) \\
= & \int_{B^{n}\left(x_{0}, 4 r / 3\right) \backslash S} F(x, \nabla h) d m(x)+\int_{S} F(x, \nabla h) d m(x) \\
\leqq & \int_{B^{n}\left(x_{0}, 4 r / 3\right) \backslash S}\left(1-(n-1) \frac{\xi^{n}}{u_{0}^{n}}\right) F\left(x, \nabla u_{0}\right) d m(x) \\
+ & \beta \frac{n^{n}}{(n-1)^{n-1}} \int_{B^{n}\left(x_{0}, 4 r / 3\right) \backslash S}|\nabla \xi|^{n} d m+\int_{S} F\left(x, \nabla u_{0}\right) d m(x) .
\end{aligned}
$$

It follows from this that

$$
(n-1) \int_{B^{n}\left(x_{0}, 4 r / 3\right) \backslash S} \frac{\xi^{n}}{u_{0}^{n}} F\left(x, \nabla u_{0}\right) d m(x) \leqq \beta \frac{n^{n}}{(n-1)^{n-1}} \int_{B^{n}\left(x_{0}, 4 r / 3\right)}|\nabla \xi|^{n} d m .
$$


Notice that $B^{n}\left(x_{0}, r\right) \cap S=\emptyset$. The condition (1.3) implies

$$
\int_{B^{n}\left(x_{0}, r\right)} \frac{\left|\nabla u_{0}\right|^{n}}{u_{0}^{n}} d m \leqq \frac{\beta}{\alpha}\left(\frac{n}{n-1}\right)^{n} \int_{B^{n}\left(x_{0}, 4 r / 3\right)}|\nabla \xi|^{n} d m \leqq c_{0} .
$$

The constant $c_{0}$ depends only on $\alpha / \beta$ and $n$. Our lemma is proved.

\section{Proof for Theorem 1.4}

Let $u_{0}$ be an extremal for the integral (1.1) and $u_{0}(x) \geqq 0$ for $x \in G$. Let $\varepsilon>0$ and define $v(x)=\log \left(u_{0}(x)+\varepsilon\right)$ for $x \in G$. The function $v$ is monotone in $G$ since $u_{0}$ is monotone by Lemma 2.3. We obtain a bound for the oscillation of $v$ from Lemma 2.2 and Lemma 2.4 :

Then we have

$$
\underset{B^{n}\left(x_{0}, r\right)}{\operatorname{Osc}}\{v\} \leqq \gamma\left(\int_{B^{n}\left(x_{0}, 3 r / 2\right)}|\nabla v|^{n} d m\right)^{1 / n} \leqq \gamma c_{0}^{1 / n} .
$$

$$
\log \left(\max u_{0}+\varepsilon\right)-\log \left(\min u_{0}+\varepsilon\right) \leqq \gamma c_{0}^{1 / n}
$$

on the set $B^{n}\left(x_{0}, r\right)$. Finally we get

$$
\max u_{0}+\varepsilon \leqq e^{\gamma c_{0}^{1 / n}}\left(\min u_{0}+\varepsilon\right) .
$$

Harnack's inequality follows if we let $\varepsilon \rightarrow 0$.

3.1. Remark. Let $f=\left(f_{1}, \ldots, f_{n}\right): G \rightarrow R^{n}$ be a quasiregular mapping; see [5], [10]. Rešetnjak [11], [12] has shown that each of the coordinate functions of $f$ minimizes a variational integral of the form (1.1). Then it follows from Theorem 1.4 that Harnack's inequality is valid for the coordinate functions. This fact has been proved earlier by Rešetnjak, who used Serrin's paper [13].

\section{References}

[1] Gehring, F. W.: Rings and quasiconformal mappings in space. - Trans. Amer. Math. Soc. 103, 1962, 353-393.

[2] Gilbarg, D., and N. S. Trudinger: Elliptic partial differential equations of second order. Springer-Verlag, Berlin-Heidelberg-New York, 1977.

[3] Granlund, S.: Strong maximum principle for a quasilinear equation with applications. Ann. Acad. Sci. Fenn. Ser. A I Math. Dissertationes 21, 1978, 1-25.

[4] John, F., and L. Nirenberg: On functions of bounded mean oscillation. - Comm. Pure Appl. Math. 14, 1961, 415-426.

[5] Martio, O., S. Rickman, and J. VäISÄLÄ: Definitions for quasiregular mappings. - Ann. Acad. Sci. Fenn. Ser. A I 448, 1969, $1-40$.

[6] Morrey, C. B.: Multiple integrals in the calculus of variations. - Springer-Verlag, BerlinHeidelberg-New York, 1966.

[7] Moser, J.: A new proof of de Giorgi's theorem concerning the regularity problem for elliptic differential equations. - Comm. Pure Appl. Math. 13, 1960, 457-468. 
[8] Moser, J.: On Harnack's theorem for elliptic differential equations. - Comm. Pure Appl. Math. 14, 1961, 577-591.

[9] Mostow, G.: Quasi-conformal mappings in $n$-space and the rigidity of hyperbolic space forms. Inst. Hautes Études Sci. Publ. Math. 34, 1968, 53-104.

[10] RešEtnjak, JU. G.: Space mappings with bounded distortion. - Siberian Math. J. 8, 1967, 466-487.

[11] ReŠETNJAK, Ju. G.: Mappings with bounded deformation as extremals of Dirichlet type integrals. - Siberian Math. J. 9, 1968, 487-498.

[12] Rešetnjak, Ju. G.: Extremal properties of mappings with bounded distortion. - Siberian Math. J. 10, 1969, 962-969.

[13] Serrin, J.: Local behavior of solutions of quasi-linear equations. - Acta Math. 111, 1964, $247-302$.

[14] Trudinger, N. S.: On Harnack type inequalities and their application to quasilinear elliptic equations. - Comm. Pure Appl. Math. 20, 1967, 721-747.

Helsinki University of Technology

Institute of Mathematics

SF-02150 Espoo 15

Finland

Received 16 May 1979

Revision received 5 October 1979 